\title{
EXPERIMENTAL DETERMINATION AND COMPUTER FITTING OF DESORPTION ISOTHERMS OF D. JOAQUINA PEARS
}

\author{
R. P. F. GUINÉ ${ }^{1}$ and J. A. A. M. CASTRO ${ }^{2}$ \\ ${ }^{1}$ Department of Food Engineering, ESAV, Polytechnic Institute of Viseu, Viseu, Portugal \\ ${ }^{2}$ Department of Chemical Engineering, The University of Coimbra, Coimbra, Portugal
}

$\mathrm{D}$

esorption isotherms at different operating temperatures provide valuable information for the design and control of drying processes. In the present work the desorption isotherms of pears are determined according to two equivalent forms of the GAB model at temperatures of 20,25 and $30^{\circ} \mathrm{C}$. These cover the range of summer average daily temperatures in Portugal, when the solar drying of pears is carried out. Two different computer fitting methodologies are used, namely, Ordinary Least Squares (OLS) and Orthogonal Distance Regression (ODR), in order to evaluate the above mentioned forms of the GAB model.

From the results obtained it is possible to conclude that the ODR fitting is clearly more adequate than the OLS technique for this kind of experimental problem. Moreover, it is advisable to exploit the full form of the GAB model instead of its simplified and restricted version. Finally, the desorption isotherms do not vary significantly in the temperature range under study.

Keywords: sorption isotherms; GAB equation; pear drying; water activity; ordinary least squares $(O L S)$; orthogonal distance regression $(O D R)$.

\section{INTRODUCTION}

The water activity strongly influences the type and intensity of the deterioration reactions that may occur in food material. Reducing the water content allows better preservation, since the material no longer constitutes an appropriate substrate for the growing of bacteria or for the occurrence of enzymatic modifications ${ }^{1,2}$. Drying is one of the most important techniques for food preservation and one of the most important operating factors is the final water content of the food; this determines the end-point of the drying process. If this final water content is too high the food stability is reduced but, on the other hand, if it is below a given optimum value, energy is wasted in the process ${ }^{3}$.

Fruits that are often dried are grapes, figs, plums, apricots and peaches, but other products have more recently gained increased importance, such as apples and pears ${ }^{4,5}$.

Success in food drying and food storage related operations (for example conditioning and packaging) strongly depends on the isothermal relationship between the water activity and water content of the food material being processed. Therefore, knowledge of such relations, often expressed in the form of sorption isotherms, at the drying and storage temperatures, is of unquestionable importance for food industry ${ }^{6}$.

All food materials are characterized by a particular sorption isotherm, correlating the water content and the relative humidity of the surrounding atmosphere, at a constant temperature. The sorption behaviour of fruits exhibits a hysteresis pattern and, thus, the corresponding isotherm shows two different curves, one for the dehydration and another for the rehydration processes which at zero humidity should form a closed loop ${ }^{7}$. Most sorption isotherms exhibit a sigmoid shape ${ }^{8}$, although some exceptions have been reported for particular food products and/or temperatures. Such isotherms provide information on how strongly the water is bound to the material ${ }^{8}$ and this is essential for the prediction of the drying and rehydration rates 9 . The information provided by sorption isotherms also enables the establishment of the ranges of water content at which the food is stable.

The aim of this paper is to investigate the sorption isotherms of a pear variety that, when dried, is highly appreciated for its quality, and whose economical importance is expected to increase in the near future in certain regions of central Portugal. Computer fitting methodologies commonly adopted in experimental programs on fruit drying will be given special attention. In particular, two equivalent models of the same isotherm will be assessed with respect to the robustness of the estimation problem. This evaluation will also be combined with the use of two different statistical techniques.

\section{EXPERIMENTAL}

The pears used in this study are of variety D. Joaquina. These are small, round products of approximately $40 \mathrm{~mm}$ 
diameter, which exhibit good drying features. Their initial water content is about $85 \%$ and the dry basis sugar content can be up to $70 \%$. When dried they are very sweet and smooth $^{10,11}$.

The experimental data for the desorption isotherms were determined using circular slices of pear $30 \mathrm{~mm}$ diameter and $3 \mathrm{~mm}$ thick, dried in a ventilated chamber (WTB-Binder) at a constant temperature. Every hour, three different samples were taken in order to measure their average water content (Mettler toledo, HG53 halogen moisture analyser) and the corresponding water activity (Rotronic hygrometer). Constant drying temperatures of 20,25 and $30^{\circ} \mathrm{C}$ were investigated, using a different set of samples for each temperature.

\section{GAB MODEL FOR SORPTION ISOTHERMS}

The Guggenheim-Anderson-de Boer (GAB) model is widely used to describe with relative accuracy the sorption isotherms of a number of food materials. The simplified form of the $\mathrm{GAB}$ equation relates the dry basis water content of the product, $W$, with the water activity, $a_{m}$ as follows ${ }^{4,8}$ :

$$
W=\frac{W_{m} C K a_{w}}{\left(1-K a_{w}\right)\left(1-K a_{w}+C K a_{w}\right.}
$$

where $W_{m}$ is the water content of the monolayer and $C$ and $K$ are temperature dependent functions. The latter have the typical form:

$$
\begin{aligned}
& C=c^{\prime} \exp \left(\frac{H_{m}-H_{n}}{R T}\right)=c^{\prime} \exp \left(\frac{\Delta H_{c}}{R T}\right) \\
& K=k^{\prime} \exp \left(\frac{H_{l}-H_{n}}{R T}\right)=k^{\prime} \exp \left(\frac{\Delta H_{k}}{R T}\right)
\end{aligned}
$$

Equations (2) and (3) enable a more complete description of the sorption isotherm since the dry basis water content of the product, $W$, is now correlated with both the water activity and the operating temperature in a single and explicit way. In these equations $H_{m}, H_{n}$ and $H_{l}$ correspond to the molar sorption enthalpies of the monolayer, multilayers and bulk liquid, respectively, and $c^{\prime}$ and $k^{\prime}$ represent entropic accommodation constants.

\section{COMPUTER FITTING PROCEDURE}

Using the data for the sorption isotherms at 20,25 and $30^{\circ} \mathrm{C}$, two basic strategies were adopted to model the process:

(1) Using the simplified version of the GAB model (see equation (1)) estimate the corresponding three parameters, where $W_{m}$ is temperature independent, and where $C$ and $K$ are temperature dependent. This procedure was repeated for all the data for each temperature in order to obtain:

- three (3) values of $W_{m}$ that should reveal some temperature invariance.

- three (3) values for $C$ and three (3) values for $K$ that should reveal an exponential variation with temperature, as indicated in equations (2) and (3). The variations were later determined by linear fitting of the triplets of $C$ and $K$ after taking the logarithm of equations (2) and (3).
Table 1. Summary of adopted fitting strategy.

\begin{tabular}{lcccc}
\hline & & \multicolumn{2}{c}{ Parameters } & \\
\cline { 3 - 4 } Case & Model & $N$ & $\begin{array}{c}\text { Estimated } \\
\text { constants }\end{array}$ & $\begin{array}{c}\text { Fitting } \\
\text { method }\end{array}$ \\
\hline 1 & $\begin{array}{c}\text { Condensed form of } \\
\text { the GAB model } \\
\text { Condensed form of } \\
\text { the GAB model } \\
\text { Expanded form of } \\
\text { the GAB model }\end{array}$ & 3 & $W_{m} C, K$ & OLS \\
3 & $W_{m} c^{\prime}, k^{\prime}, \Delta H_{C}, \Delta H_{K}$ & ODR \\
\hline
\end{tabular}

(2) Using the full form of the GAB model (represented by the three simultaneous equations (1), (2) and (3)) directly estimate five parameters, namely, $W_{m}, c^{\prime}, k^{\prime}$, $\Delta H_{c}$ and $\Delta H_{k}$. This procedure was applied to all data (i.e. for all three temperatures).

In this work, a different fitting technique, the ODR method, will be evaluated against the ordinary least squares (OLS); the latter fitting method is most often used in this type of study ${ }^{4}$.

The orthogonal distance regression (ODR) method is one of the so called EVM (errors-in-variables) methods in which unknown errors are taken into account in both dependent and independent variables. The problem can be formulated as follows: consider a set of data $\left(x_{i}, y_{i}\right), i=1, \ldots, n$, where $y_{i}$ is supposed to be a non-linear function of $x_{i}$ and a set of parameters $\beta_{k}$. If both $x_{i}$ and $y_{i}$ contain unknown errors $\delta_{i}$ and $\varepsilon_{i}$, then the observed value of $y_{i}$ satisfies $^{12}$ :

$$
y_{i}+\varepsilon_{i}=f_{i}\left(x_{i}+\delta_{i} ; \beta_{k}\right) ; \quad i=1, \ldots, n
$$

for some set of values $\beta_{k}, k=1, \ldots, n_{p}$. As a result of unknown errors in both the dependent and independent variables, the ODR method finds the best solution of the estimation problem by minimizing the $n$ orthogonal distance from the curve $f(x ; \beta)$ to all data points, i.e. ${ }^{12}$ :

$$
\min _{\beta} \sum_{i=1}^{n}\left(\delta_{i}^{2}+\varepsilon_{i}^{2}\right)
$$

This is in clear contrast with the conventional OLS technique where unknown errors, $\delta_{i}$, in the independent variables, $x_{i}$, are never taken into account.

When the expanded form of the GAB model is used to estimate the afore mentioned parameters, the function is of the form:

$$
W=f\left(a_{w}, T ; \beta_{k}\right)
$$

where $W$ represents the dependent variable, and $a_{w}$ and $T$ the two independent variables that are affected by non-zero unknown measurement errors of different statistical means. In this case, $\varepsilon_{i}$ refer to errors in $W$, and $\delta_{i}$ express the residues in the two independent variables: $a_{w}$ and $T$.

The software package used to compute the parameters is ODRPACK, developed by the Center for Computing and Applied Mathematics of the National Institute of Standards and Technology (USA) ${ }^{12}$. Table 1 details the fitting strategy that was used in this work. It can be seen that the ODR was employed for both forms of the GAB model, and the OLS technique was only used for comparison purposes. 


\section{RESULTS AND DISCUSSION}

The results of the parameter estimation carried out using case- 1 are shown in Table 2. Based on the high values of the sum of the square of the residuals and residual standard deviation, it is clear that the estimation is of poor quality. Moreover, the optimal estimate for the supposedly temperature independent parameter, $W_{m}$, shows a remarkable sensitivity to temperature which is also a indication of the poor quality of the estimation.

Table 3 shows the results of the estimation using the ODR technique applied to the same simplified form of the GAB model. As it can be seen, the values of the sum of the square of the errors and the residual standard deviation are much smaller than those characterizing the OLS fitting procedure (see Table 3). In spite of this clear improvement in the statistical analysis of the problem, there is still an unquestionable sensitivity of $W_{m}$ to temperature; this is to a much less extent than in the ODR procedure.

The better performance of the ODR, as shown by the results of this preliminary study, is not surprising because it is well known that ordinary least squares is based on the assumption that there are no unknown measurement errors in the independent variables. This means that it can only be applied to problems with errors in the dependent variables, and this is undoubtedly not the case in the present study. In fact, taking a closer look at the GAB model the independent variable $a_{m}$, the water activity, is experimentally measured with some kind of uncertainty. This fact is corroborated by the higher values of the sum of square of the errors associated with $a_{w}$ in comparison to those of $W$, the dependent variable. Error analysis is appropriate to confirm this.

Table 2. Estimation of the simplified form of the GAB model using OLS (case-1).

\begin{tabular}{lccc}
\hline & \multicolumn{3}{c}{ Temperature } \\
\cline { 2 - 4 } Parameter & $20^{\circ} \mathrm{C}$ & $25^{\circ} \mathrm{C}$ & $30^{\circ} \mathrm{C}$ \\
\hline$W_{m}$ & 0.287 & 1.604 & 0.179 \\
$C$ & 0.149 & 0.026 & 0.709 \\
$K$ & 0.986 & 0.949 & 0.997 \\
$N$ of observations & 35 & 28 & 53 \\
Sum of square & 3.656 & 1.915 & 4.999 \\
$\quad$ errors & & & 0.3162 \\
$\quad$ Residual standard & 0.3350 & 0.2768 & \\
$\quad$ deviation & & & \\
\hline
\end{tabular}

Table 3. Estimation of the simplified form of the GAB model using ODR (case-2).

\begin{tabular}{lccc}
\hline & \multicolumn{3}{c}{ Temperature } \\
\cline { 2 - 4 } Parameter & $20^{\circ} \mathrm{C}$ & $25^{\circ} \mathrm{C}$ & $30^{\circ} \mathrm{C}$ \\
\hline$W_{m}$ & 0.158 & 0.218 & 0.211 \\
$C$ & 0.409 & 0.266 & 0.399 \\
$K$ & 1.005 & 0.991 & 0.993 \\
$N$ of observations & 35 & 28 & 53 \\
$\Sigma \delta^{2}\left(a_{w}\right)$ & 0.009675 & 0.006232 & 0.02562 \\
$\Sigma \varepsilon^{2}(W)$ & 0.001169 & 0.000975 & 0.01385 \\
Sum of square of errors & 0.010844 & 0.007207 & 0.03947 \\
Residual standard deviation & 0.01841 & 0.01698 & 0.02810 \\
\hline
\end{tabular}

In Figure 1 (graphs (a) and (b)) the quantifying residues associated with both variables, $a_{w}$ and $W$, obtained with the ODR fitting, are plotted against the corresponding variable, for the three temperatures. Comparing graphs (a) and (b), it is noticeable that the errors associated with the independent variable, $a_{w}$, are more significant than the errors associated with the dependent variable, $W$, corroborating the earlier suggestion.

The residues for $a_{w}$ and $W$ are represented in frequency mode in Figure 2, and the corresponding mean values and standard deviations are summarized in Table 4 . From this it is possible to confirm that the mean values of the residues of $a_{w}$ are of the order $10^{-2}$ and those of $W$ are of one order lower, i.e. $10^{-3}$. This difference is even more relevant when taking into account that $a_{w}$ varies between a minimum of zero and a maximum of one, while $W$ can vary from 0 to infinity.

These particular statistical properties of the experimental data are an indication that some alternative curve fitting method should be used instead of the ordinary least squares (OLS) because the assumptions on which it is based are not satisfied. This is, however, very appropriate for hand-

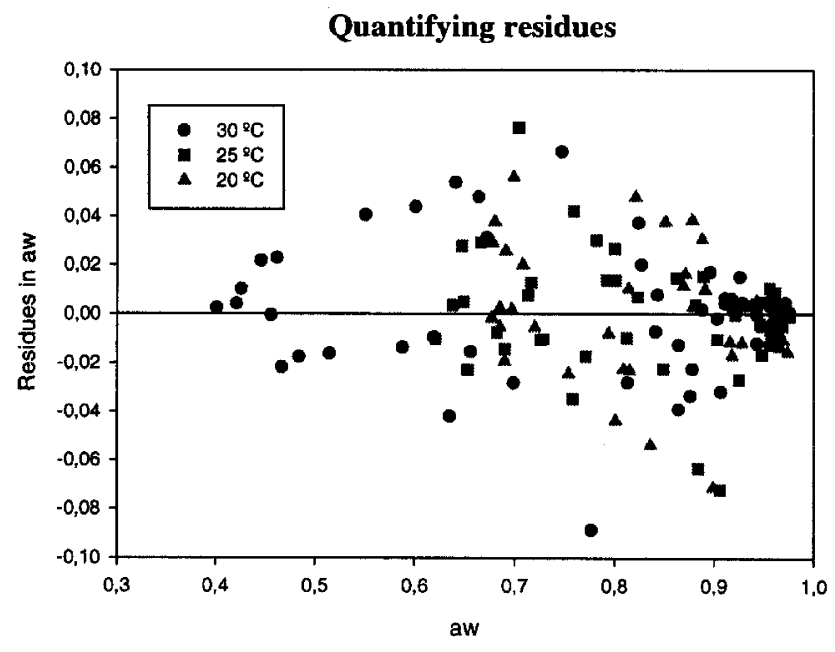

(a)

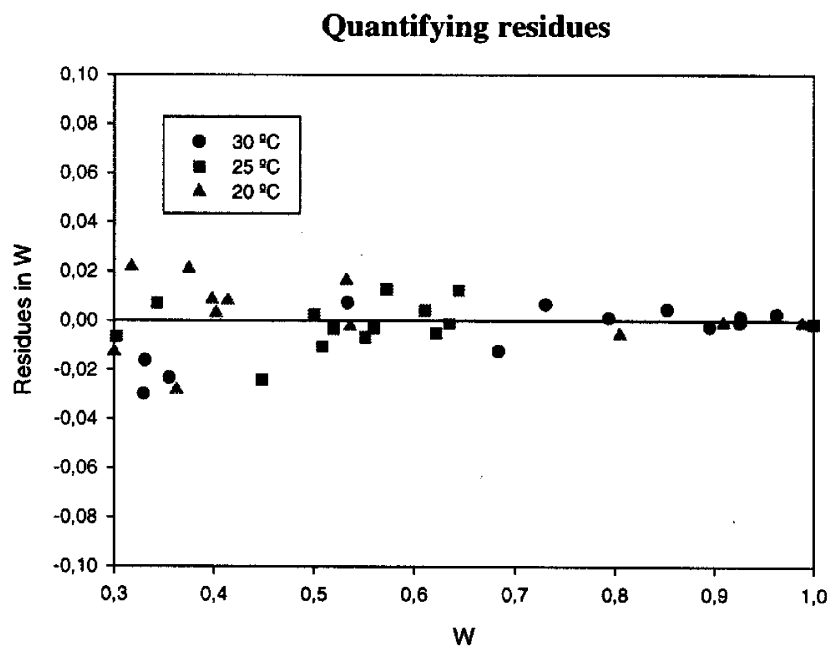

(b)

Figure 1. Residues associated with: (a) the independent variable, $a_{w}$; (b) the dependent variable, $W$. 

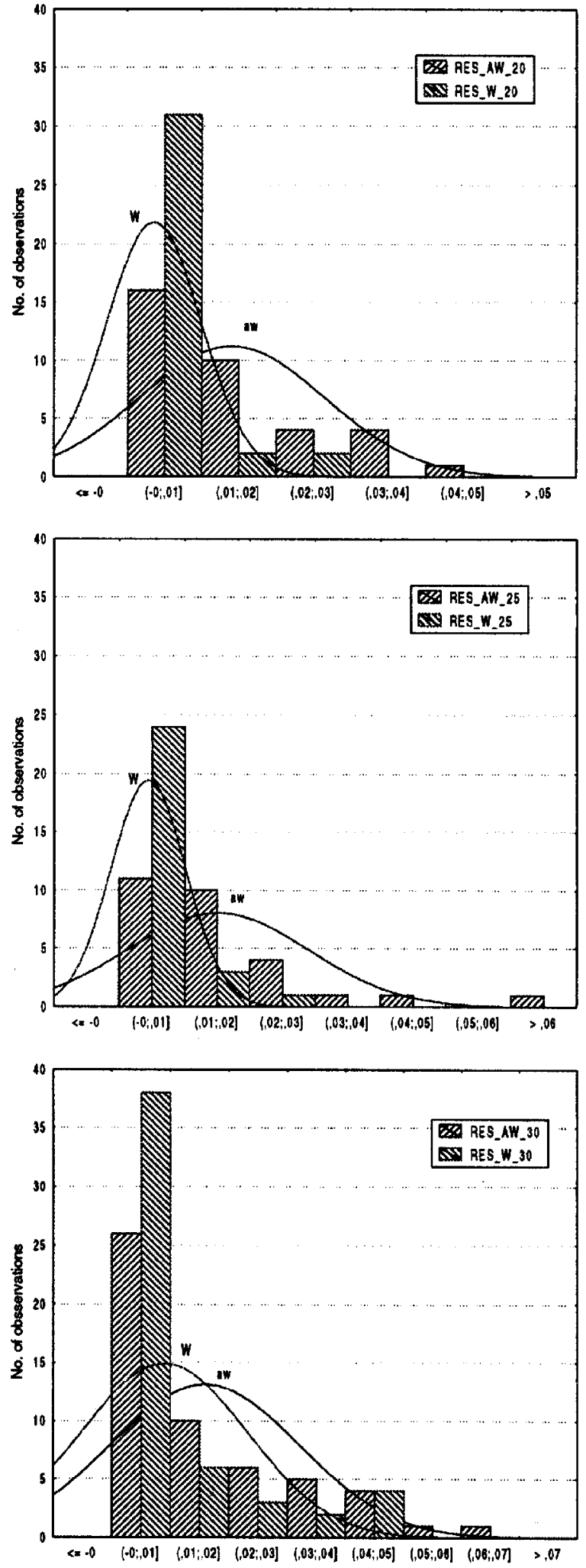

Figure 2. Normal distribution of the residues in $a_{w}$ and $W$ for the three temperatures studied.

ling with an EVM method and especially with the ODR algorithm.

Due to the great potential of ODR for multi-parameter estimation with errors in all variables, the data obtained
Table 4. Statistical analysis of the residuals.

\begin{tabular}{clccc}
\hline & & $T=20^{\circ} \mathrm{C}$ & $T=25^{\circ} \mathrm{C}$ & $T=30^{\circ} \mathrm{C}$ \\
\hline $\begin{array}{c}\text { Residues } \\
\text { in } a_{w}\end{array}$ & Mean value & 0.01400 & 0.01510 & 0.01590 \\
& $\begin{array}{c}\text { Standard } \\
\text { deviation, } \sigma_{a_{w}}\end{array}$ & 0.01240 & 0.01390 & 0.01610 \\
$\begin{array}{c}\text { Residues } \\
\text { in } W\end{array}$ & $\begin{array}{c}\text { Mean value } \\
\text { Standard } \\
\text { deviation, } \sigma_{\mathrm{W}}\end{array}$ & 0.00353 & 0.00443 & 0.00897 \\
& & 0.00640 & 0.00576 & 0.01420 \\
\hline
\end{tabular}

experimentally at three temperatures will be processed as a single set of 116 triplets $\left(W, a_{w}, T\right)$ against the full form of the GAB model (with five parameters, see Table 1). The results of this full parameter estimation are summarized in Table 5.

The statistical treatment of the residuals for case 3 are presented in Figure 3 and Table 6. This confirms that the errors in one of the independent variables $\left(a_{w}\right)$ is clearly higher than the dependent variable $(W)$. The results also confirm that the other independent variable $(T)$ is practically negligibly affected by errors, as expected.

Since the proposed method of estimation (full form of the GAB model and ODR algorithm) enables a more robust statistical processing of the data, it is possible, using the optimal parameter estimates shown in Table 5, to calculate the sorption isotherms of the pears at a temperature range slightly wider than that in the experimental programme. These are illustrated in Figure 4 for temperatures of 20,40 and $60^{\circ} \mathrm{C}$. As can be seen, the differences between the isotherms are not significant. However, the slight variation in the isotherms shows that increasing the temperature gives rise to a higher isotherm. This behaviour is not usual; at constant moisture content, an increase in temperature enhances water activity quite considerably to most solid fruits ${ }^{4,13}$. Nevertheless, the results of this study are as expected because this fruit is mainly constituted of sugars (up to $70 \%$ dry basis) and these are reported to exhibit an opposite behaviour when compared to most solid food products, due to the solution of solids in water ${ }^{6,14}$.

\section{CONCLUSIONS}

A new methodology to fit the experimental data for the desorption isotherms of D. Joaquina pears at 20, 25 and

Table 5. Summary of the results for the optimal estimation of the full form of GAB model with the ODR algorithm.

\begin{tabular}{lc}
\hline Parameters & Optimal estimate \\
\hline$W_{m}$ & 0.1760 \\
$c^{\prime}$ & 6.389 \\
$k^{\prime}$ & 1.0125 \\
$\Delta H_{C}$ & -6751 \\
$\Delta H_{K}$ & -29 \\
& \\
$N$ of observations $\quad$ Statistical information & 116 \\
$\Sigma \delta^{2}\left(a_{w}, T\right)$ & 0.04635 \\
$\Sigma \varepsilon^{2}(W)$ & 0.01809 \\
Sum of square of errors & 0.06444 \\
Residual standard deviation & 0.02420 \\
\hline
\end{tabular}




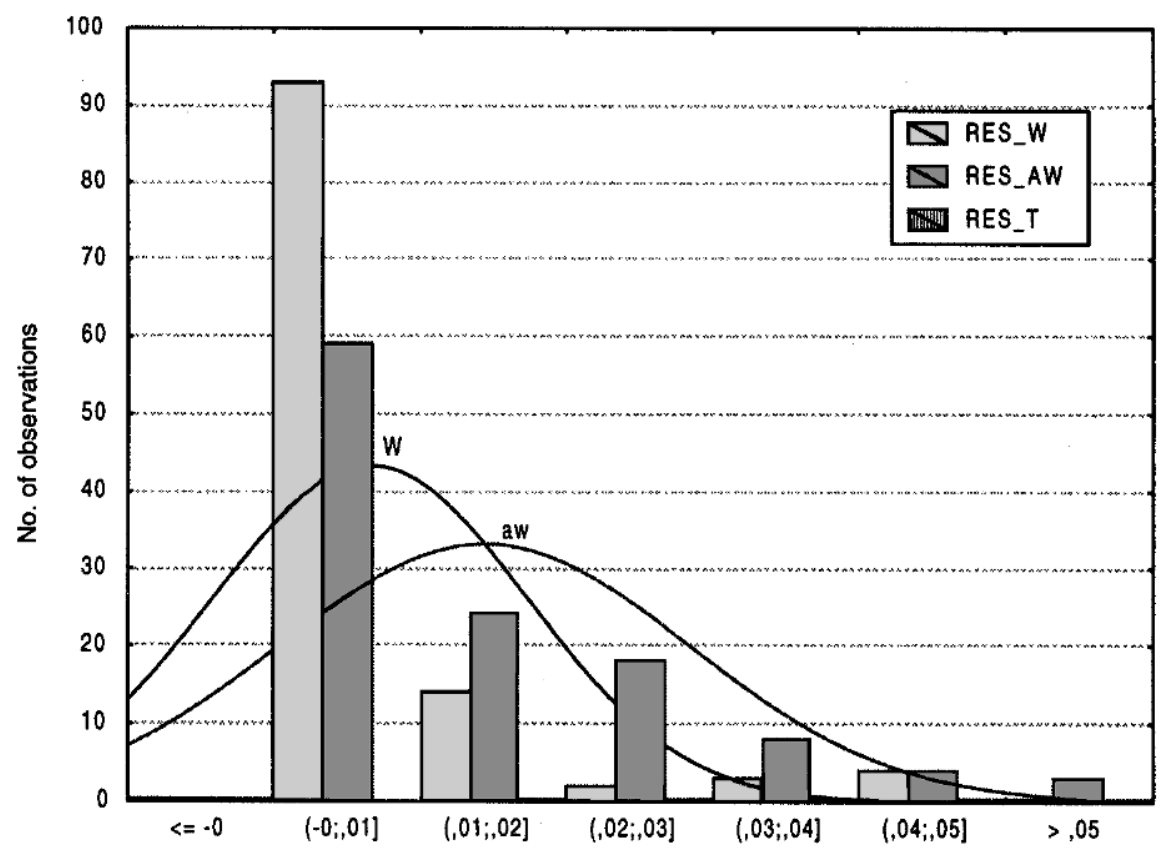

Figure 3. Normal distribution of the residues for $W, a_{w}$ and $T$.

Table 6. Statistical analysis of the residuals for the three variables.

\begin{tabular}{llr}
\hline & Independent variables & \\
Residues in $a_{w}$ & Mean value & $14.4 \times 10^{-3}$ \\
& Standard deviation, $\sigma a_{w}$ & $14.0 \times 10^{-3}$ \\
Residues in $W$ & Mean value & $0.008 \times 10^{-3}$ \\
& Standard deviation, $\sigma a_{w}$ & $0.011 \times 10^{-3}$ \\
& $\quad$ Dependent variable & \\
Residues in $T$ & Mean value & $6.5 \times 10^{-3}$ \\
& Standard deviation, $\sigma_{\mathrm{T}}$ & $10.7 \times 10^{-3}$ \\
\hline
\end{tabular}

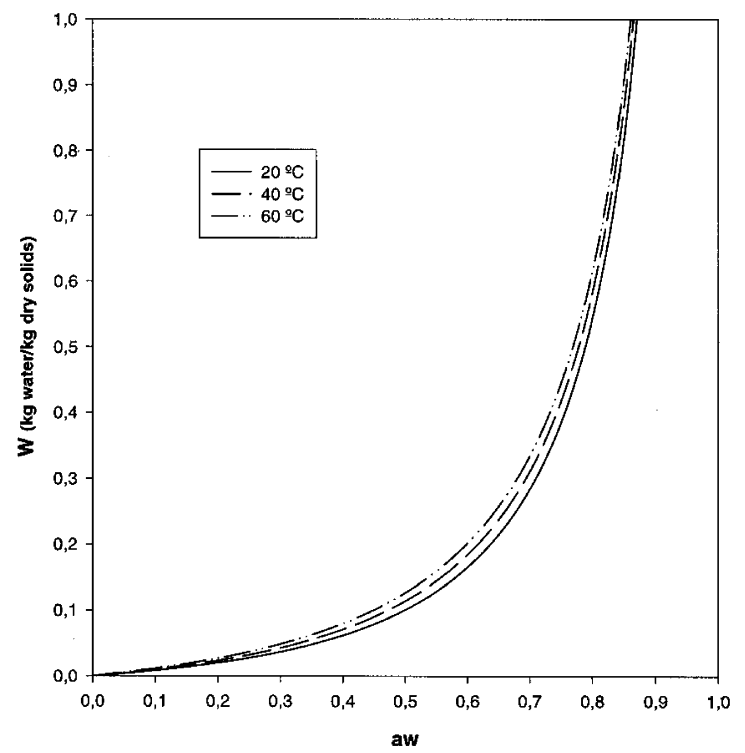

Figure 4. GAB model desorption isotherms for pears at 20,40 and $60^{\circ} \mathrm{C}$. $30^{\circ} \mathrm{C}$ has been proposed and evaluated. The ordinary least squares (OLS) fitting method was shown to not adequately estimate the $\mathrm{GAB}$ equation parameters, since it assumes that there are no errors in the independent variables, which is not the case in this study. As an alternative, the orthogonal distance regression (ODR) has been assessed and its performance was shown to be unquestionably superior.

The results reveal that, for the temperature range between 20 and $30^{\circ} \mathrm{C}$, there is no meaningful sensitivity, i.e. the equilibrium between the relative humidity of the air, or its water activity, and the pear water content does not significantly change with temperature. This is of particular importance as the solar drying of these pears is carried out at open air and, therefore, is subject to natural temperature variations of this magnitude. However, if industrial processing were to be sought in the future, there would be important gains if it were possible to widen the range of operating temperatures, and if an optimization scheme were applied to take full advantage of this work.

\section{NOMENCLATURE}

$\begin{array}{ll}a_{w} & \text { water activity } \\ C & \text { temperature dependent function }\end{array}$

$c^{\prime} \quad$ entropic accommodation constant

$H_{m} \quad$ molar sorption enthalpy of the monolayer, $\mathrm{J} \mathrm{mol}^{-1}$

$H_{n} \quad$ molar sorption enthalpy of the multilayers, $\mathrm{J} \mathrm{mol}^{-1}$

$H_{l} \quad$ molar sorption enthalpy of the bulk liquid, $\mathrm{J} \mathrm{mol}^{-1}$

$i \quad$ integer variable

$K \quad$ temperature dependent function

$k^{\prime} \quad$ entropic accommodation constant

$n$ number of data points

$R \quad$ constant of gases, $\mathrm{J}_{\mathrm{mol} \mathrm{K}} \mathrm{K}^{-1}$

$T \quad$ temperature, $\mathrm{K}$

$W \quad$ dry basis water content, $\mathrm{kg}^{-1}$

$W_{m} \quad$ water content of the monolayer, $\mathrm{kg}^{-1}$

$x \quad$ dependent variable

$y \quad$ independent variable 
Greek symbols

$\beta \quad$ set of parameters

$\delta \quad$ error associated with the independent variable

$\Delta H_{c} \quad$ enthalpy difference for $C(T)$ function, $\mathrm{J} \mathrm{mol}^{-1}$

$\Delta H_{k} \quad$ enthalpy difference for $K(T)$ function, $\mathrm{J} \mathrm{mol}^{-1}$

$\varepsilon \quad$ error associated with the dependent variable

\section{REFERENCES}

1. Mabanda, P. S., 1997, Drying Technology, 15(1): 117-136.

2. Salgado, M. A., Lebert, A., Garcia, H. S., Muchnik, J. and Bindenet, J. J., 1994, Drying Technology, 12(3): 685-696.

3. Vázquez-Uña, G., Chenlo-Romero, F. and Moreire-Martínez, R., 2001, The 8th Inter Conf Chem Eng, pp. 1077-1081.

4. Kiranoudis, C. T., Tsami, E., Maroulis, Z. B. and Marinos-Kours, D., 1997, Drying Technology, 15(5): 1399-1418.

5. Chiang, W. C. and Peterson, J. N., 1987, Drying Technology, 5(1): $25-49$.

6. Vázquez-Uña, G., Chenlo-Romero, F. and Moreire-Martínez, R., 2001, The 8th Inter Conf Chem Eng, 1071-1076.

7. Watt, I. C., 1986, In: Physical Properties of Foods, R. Jowitt et al. (eds) (Elsevier Applied Science Publishers, Great Yarmouth, USA), pp. $27-42$.

8. Weisser, H., 1986, In: Food Engineering and Process Applications: Transport Phenomena, M. L. Maguer, and P. Jelen, (eds) (Elsevier Applied Science Publishers, Alberta, USA), Vol. 1, pp. 189-200.
9. Fellows, P., 1994, Tecnologia del Procesado de los Alimentos: Princípios y Practicas (Acribia, Zaragoza, Spain).

10. Guiné, R. P. F. and Castro, J. A. A. M., 2001, The 8th Inter Conf Chem Eng, pp. 1189-1193.

11. Guiné, R. P. F. and Castro, J. A. A. M., 2001, $5^{\circ}$ Encontro de Química dos Alimentos, pp. 504-506.

12. Boggs, P. T., 1992, User's Reference Guide for ODRPACK Version 2.01. Software for Weighted Orthogonal Distance Regression (National Institute of Standards and Technology, Gaithersburg, USA).

13. Johnson, P. N. T. and Brennan, J. G., 2000, J Food Eng, 44: 79-84.

14. Maroulis, Z. B., Tami, E., Marinos-Kouris, D. and Saravacos, G. D., 1988, J Food Eng, 7: 63-78.

\section{ADDRESS}

Correspondence concerning this paper should be addressed to Professor R. P. F. Guiné, Department of Food Engineering, Polytechnic Institute of Viseu, Escola Superior Agraria de Viseu, Campus Politecnico, Repeses, 3504-510 Viseu, Portugal.

E-mail: raquelguine@esav.ipv.pt

The manuscript was received 27 November 2001 and accepted for publication after revision 9 May 2002. 